\title{
Real-time integrity monitoring for a wide area precise positioning system
}

\author{
Yuechen Wang ${ }^{*}$ (D) and Jun Shen
}

\begin{abstract}
The wide area precise positioning system (WAPPS) is a high-precision positioning system based on a global navigation satellite system. Using a GEO satellite or a communication network, it provides users, in its service area, with real-time satellite orbit, clock, and other corrections. Users can achieve centimeter-level static positioning or decimeter-level kinematic positioning by precise point positioning. With the demands for applications of both high-precision and safety of life in real time, WAPPS is facing urgent needs to improve its service integrity. This study presents a realtime integrity monitoring approach for WAPPS. Using dual-frequency ionosphere-free corrections of GPS and BDS, along with monitor station data, related error models are established and the integrity monitoring is achieved, based on the analysis of satellite corrected residuals. In addition, satellite faults are simulated for performance verification. The results show that the algorithm can monitor both step and drift faults effectively and alert users in time.
\end{abstract}

Keywords: Wide area precise positioning system, GPS correction, BDS correction, Integrity monitor

\section{Introduction}

As an important wide-area differential systems associated with global navigation satellite systems (GNSS), the Wide Area Precise Positioning System (WAPPS) transmits the satellite's real-time precise orbit and clock corrections through a GEO satellite or a communication network to improve positioning and navigation accuracy. Users receive both GNSS and WAPPS signals and achieve high-precision positioning by precise point positioning (PPP) (Shi et al. 2009). With the advantages such as lower density of stations, wider service areas, and simpler user terminals, WAPPS has been commonly used in maritime, geological mapping, precision agriculture, and other applications (Niu et al. 2007). As automotive and unmanned aerial vehicle (UAV) applications emerge, WAPPS is facing the needs on safety of life and needs to improve integrity, continuity, and availability based on high-precision services. In particular, the integrity refers to the ability to alert users in time when the service fails to meet the required performance, which is

*Correspondence: yc.wang_dh@unistrong.com

Beijing UniStrong Science and Technology Corporation Limited, Beijing 100176, China directly related to the safety of real-time dynamic applications. There are three parameters in integrity monitoring: integrity risk, alert limit and time-to-alert.

Since the end of last century, WAPPS and PPP have been studied widely. Firstly, related studies on principles and algorithms based on GPS have been conducted, which prove that the service can achieve high-precision positioning results in real time effectively (Zumberge et al. 1997; Kouba and Hérous 2001). Then, the research on the integer ambiguity resolution of carrier phase has been carried out through different methods, which provide more precise and stable services in real time (Collins 2008; Ge et al. 2008; Laurichesse et al. 2008). In recent years, with the construction of BDS, the calculation and application of the BDS corrections, which can shorten the convergence time of services (Qu et al. 2013; Cui et al. 2015), has been researched and achieved. These WAPPS and PPP studies mainly focus on the performance improvement of positioning accuracy and convergence time, while the integrity of services has not been taken into consideration.

In terms of integrity, it is mainly researched and applied in civil aviation applications, including the Airborne-Based Augmentation System (ABAS) (Parkinson 
and Axelrad 1988; Brown 1992; Blanch et al. 2010), the Satellite-Based Augmentation System (SBAS) (Tsai 1999; Blanch 2003; Todd Walter et al. 2012), and the GroundBased Augmentation System (GBAS) (Braff 1997; Sayim 2003; Luo et al. 2003). These studies mainly aim at the integrity monitoring based on pseudo range, which are realized by using method of redundant data checking, residual statistics and error overbound. However, the integrity monitoring for high-precision positioning with carrier phase has not been involved in these studies.

In this study, a preliminary WAPPS integrity monitoring is achieved, using real-time corrections generated through an in-house developed system. First, the definition of the WAPPS integrity monitoring is put forward. Then, an algorithm is designed to realize the monitoring using real-time GPS/BDS corrections and ground station data. Finally, the algorithm performance is verified by the simulation of specific faults on corrections.

\section{Analysis of WAPPS correction}

The analysis of the GPS and BDS correction performance is carried out as an input for the integrity monitoring, which includes the accuracy of both the orbit and clock corrections, as well as the accuracy of user positioning.

A data set from the Deutsches GeoForschungsZentrum (GFZ) is used to evaluate the correction accuracy. The evaluation parameter is named dSISE, which is similar to the GNSS signal-in-space error (SISE) (Chen et al. 2015;
US Department of Defense 2008) and indicates the error between the corrections used and GFZ's products, as expressed in Eq. (1). R, A, and C represent, the orbit error in radial, along, and cross directions, respectively, and $\mathrm{T}$ represents the satellite clock error of two types of products, which has eliminated the influence of inconsistency in time and frequency references.

$$
\begin{aligned}
& d S I S E_{G P S}=\sqrt{(0.980 R-T)^{2}+0.141 \cdot\left(A^{2}+C^{2}\right)} \\
& d S I S E_{B D S \_G / I}=\sqrt{(0.992 R-T)^{2}+0.088 \cdot\left(A^{2}+C^{2}\right)} \\
& d S I S E_{B D S \_M}=\sqrt{(0.980 R-T)^{2}+0.141 \cdot\left(A^{2}+C^{2}\right)}
\end{aligned}
$$

Figure 1 shows the correction accuracy during the period from September 1-30, 2018. The results of GPS and BDS are presented at the top and bottom of the figure. The blue and red histograms represent dSISE's mean and variance, respectively. The mean is less than $1 \mathrm{~m}$ for GPS and approximately $1.1 \mathrm{~m}$ for BDS. As for the standard deviation of dSISE, that of GPS is less than $0.2 \mathrm{~m}$ and that of BDS is about $0.4 \mathrm{~m}$, which is sufficient to support a real-time PPP.

Figure 2 shows the typical dual-frequency kinematic PPP result using WAPPS corrections. The graphic at the top shows the results of position, where the blue, green, and red point sequences represent the positioning errors in the east, north, and up direction, respectively. The chart at the bottom shows the number of visible satellites. It can be observed that the stable accuracy is
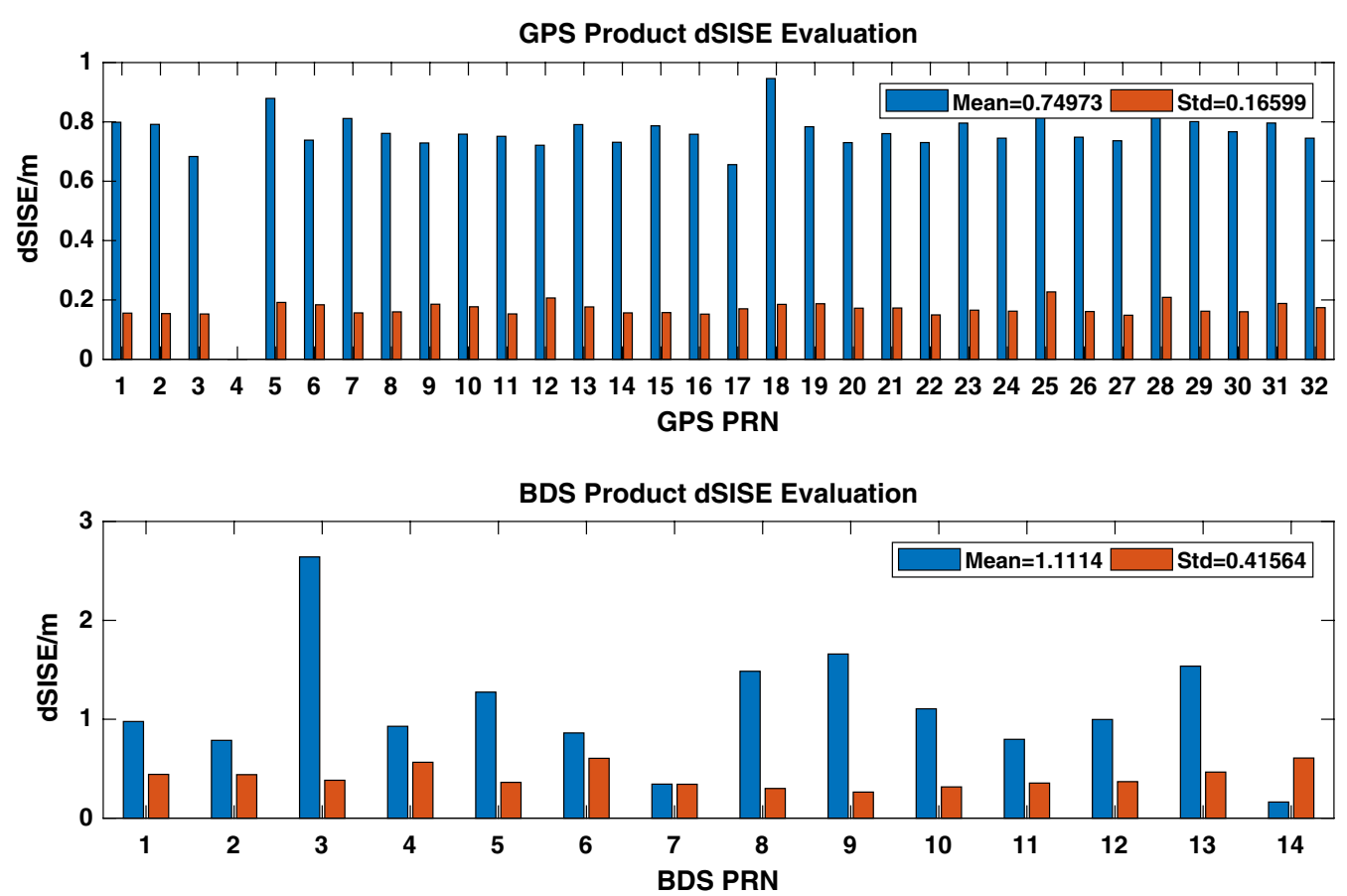

Fig. 1 Accuracy statistics of GPS and BDS corrections of WAPPS 

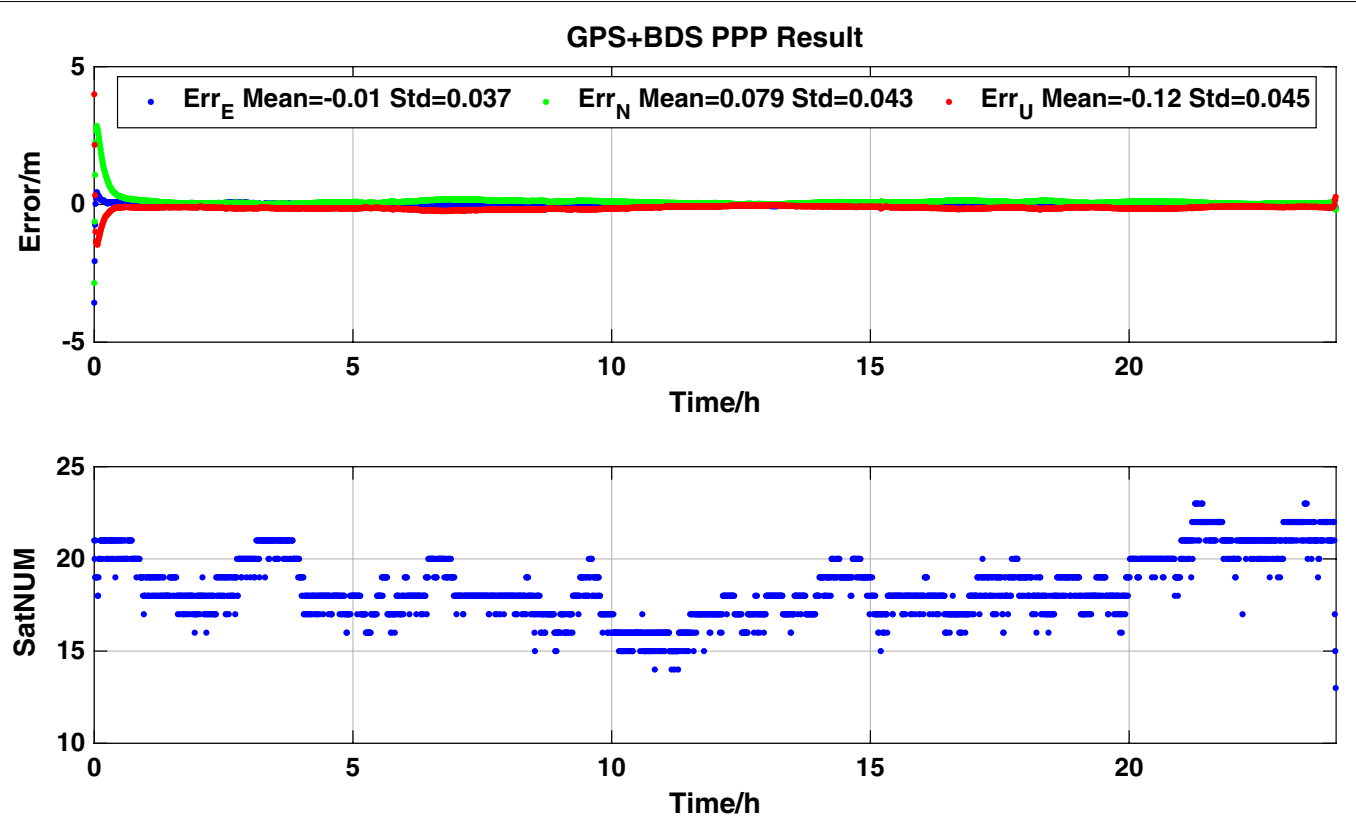

Fig. 2 Typical result of dual-frequency kinematic PPP using WAPPS

approximately $0.2 \mathrm{~m}$, with a convergence time of approximately $30 \mathrm{~min}$, which can meet the needs of most of users.

\section{Integrity monitoring for WAPPS Definition of integrity monitoring}

Different from a SBAS used in civil aviation, integrity monitoring for WAPPS has its own particularity. The research of integrity monitoring in this work is mainly based on the following definitions:

- The integrity monitoring for WAPPS mainly focuses on the correction of the services; there is no monitoring on the satellite without corrections.

- WAPPS provides high-precision services by correcting the pseudo range and carrier phase measurements; hence, it is necessary to monitor the performance of corrections on both the pseudo range and carrier phase simultaneously. Based on the performance of our research operating system, the initial requirement of the miss alert rate is $10^{-3}$, the false alert rate is $10^{-5}$, and the time-to-alert is $10 \mathrm{~s}$. Moreover, with clarity of needs, these parameters may be changed in a future study.

- WAPPS mainly uses a dual-frequency ionospherefree (DFIF) measurement to provide services; thus, the ionosphere anomaly is not considered here.

- The user segment anomalies, such as carrier phase cycle slip, need to be guaranteed by the receiver and have not been considered in WAPPS integrity monitoring.

Based on the definition above, the correction threat factors in WAPPS are shown below, which can be summarized into two types of integrity fault modes: a step fault and a slow drift fault.

- An orbit correction fault. In the precise orbit determination and prediction, there may be a step error caused by unsmooth results between the orbit solutions and a slow drift error caused by anomalous extrapolation. Satellite maneuvering will lead to the step error, which need to be monitored if there is no alert in the GNSS service.

- A clock correction fault. Satellite clock's faults such as rapid jumping and aging drift may occur. The time series filter of the clock correction estimation cannot reflect the real-time clock error effectively, resulting in step or slow drift faults.

- Bit errors in broadcasting. The high rate of data transmission and low power of signal may lead to bit error of corrections, which can result in step fault.

There are many threat factors in WAPPS, which might cause abnormal deviation on the user's observation measurements and affect positioning integrity eventually. Therefore, the WAPPS integrity monitoring will focus on the corrected measurement residuals, such as pseudo range and carrier phase. The realization 
process includes data analysis and modeling, parameter and process design, calculation, and verification.

\section{Statistics on corrected residuals}

To realize integrity monitoring, $1 \mathrm{~s}$ sampling corrected residuals of the DFIF pseudo range and carrier phase are analyzed to complete the integrity risk modeling. The tropospheric error is mitigated by the model. due to ground stations are not currently equipped with atomic clocks, the receiver clock error is calculated by using broadcast ephemeris and smoothed in real time. To avoid an abnormity in the calculation of the receiver clock, the fault detection is accomplished by using the precise coordinates of stations. In the future, it is necessary to equip stations with atomic clocks to achieve a more reliable monitoring and to reduce the integrity risk rate.

Data of 10 stations in the period from September 16, 2018 to September 22, 2018 are used, and quantilequantile plots of all satellites between the normalized WAPPS corrected residual distribution and the standard Gauss distribution are shown in Figs. 3 and 4, represented in blue.

With the statistics on corrected residuals, the following conclusions can be drawn.
- The distributions of both the corrected pseudo range and the carrier phase residuals are consistent with the Gaussian distribution in the central area. However, in the tail distribution, in which integrity monitoring is concerned, the quantile of residual distribution is smaller than the quantile of the Gaussian distribution. This indicates that the standard Gaussian distribution can be used to complete the over bound of the tail of the residual, so as to reduce the probability of integrity miss alert risk.

- The normalized corrected pseudo range residual's mean is zero and the standard deviation is $0.6-0.9 \mathrm{~m}$, which can be bounded by a Gauss well.

- The normalized corrected carrier phase residual's standard deviation is $0.5-0.8 \mathrm{~m}$ and can also be bounded by Gauss. In addition, due to the inaccuracy of the ambiguity fixing, there are also a few anomalies in the tail.

- The pseudo range residual distributions of a few satellites are different from those of most others because of the differences in performance of the satellite and station, measurement noise, model's corrected accuracy, and performance of the station clock, identi$\mathrm{cal}$, whereas the distributions of phase residual are almost the same. The assumption of an independent and identical distribution will be used in the moni-

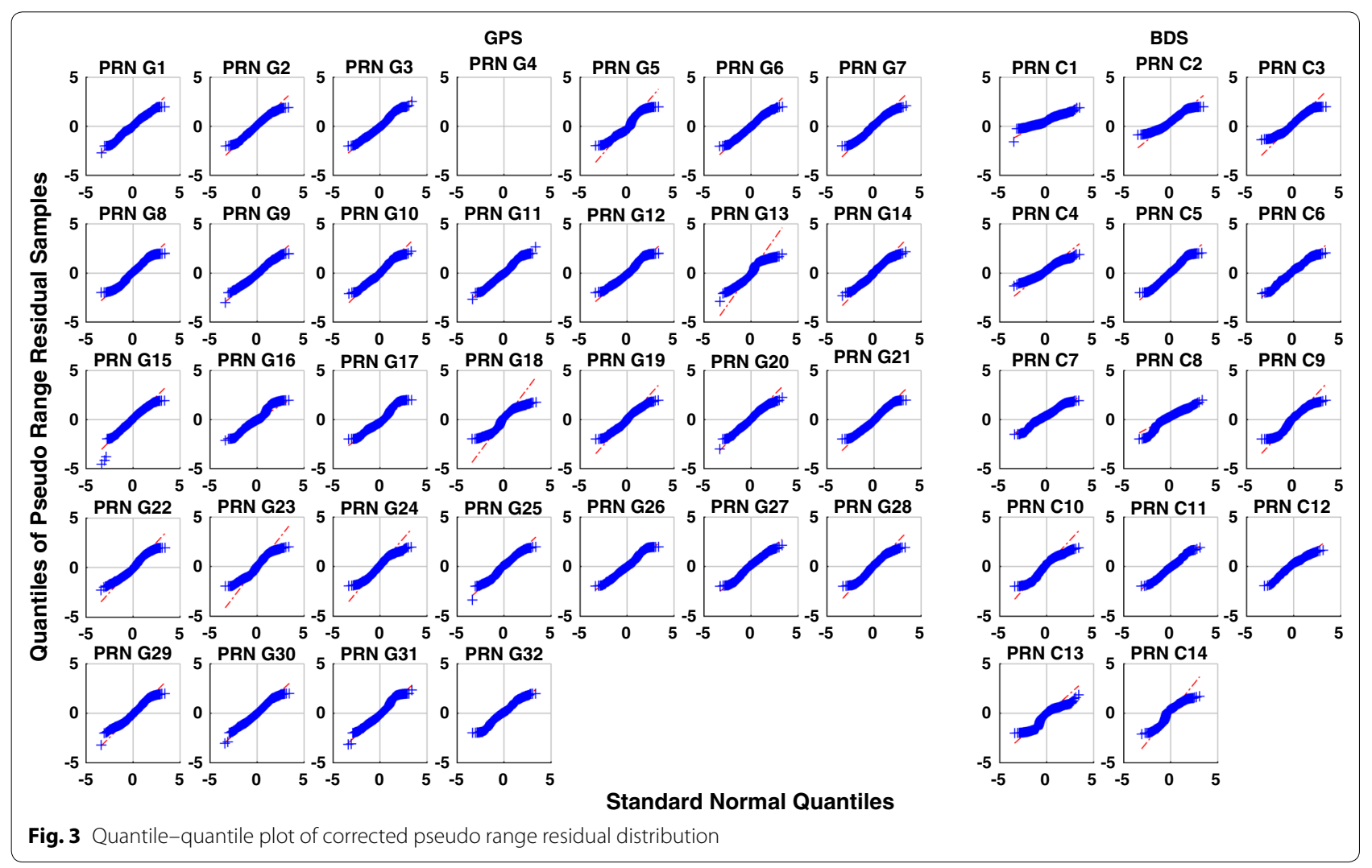



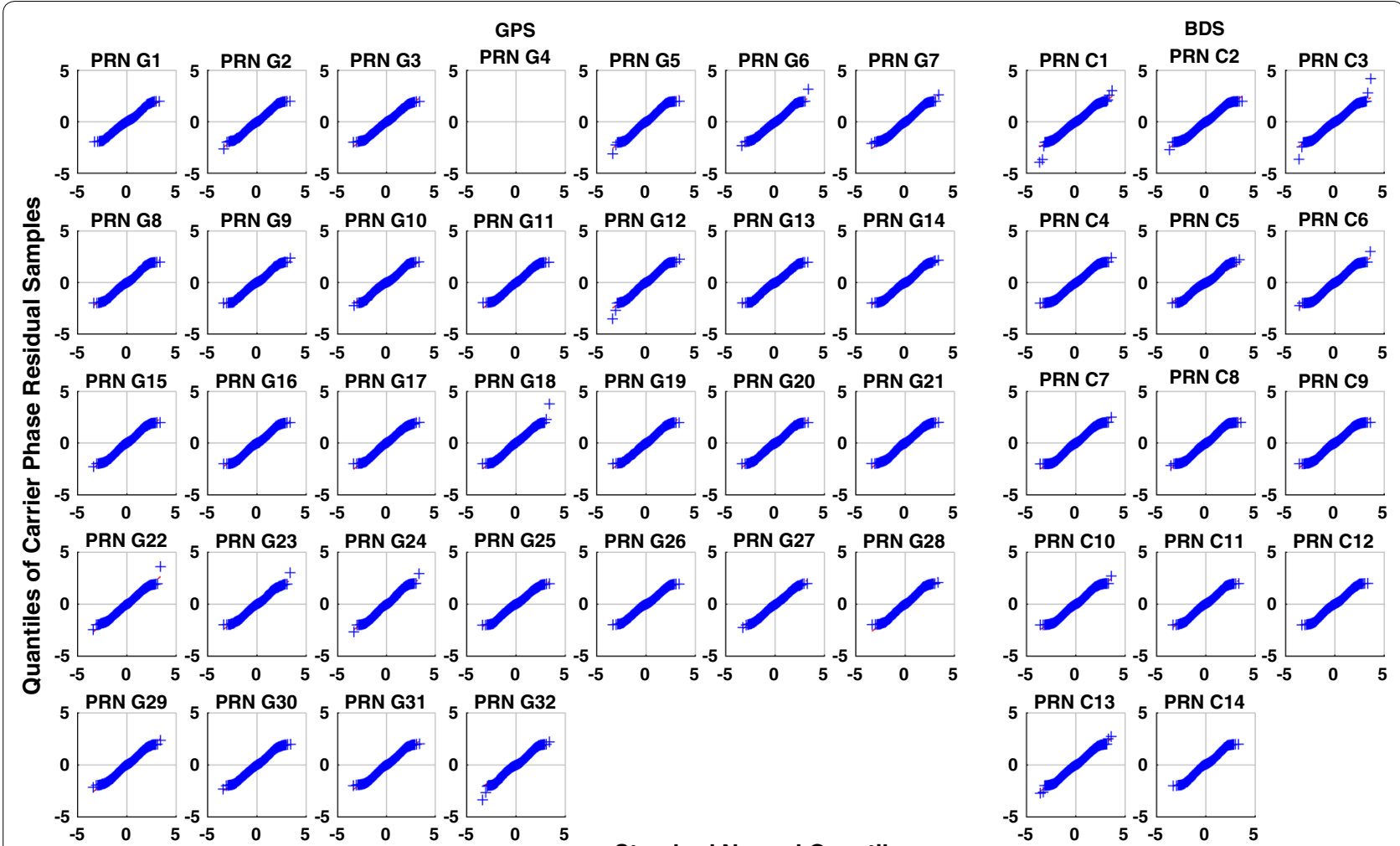

Fig.4 Quantile-quantile plot of corrected carrier phase residual distribution

toring, and a relevant test will be used to verify the performance.

\section{Design of integrity monitoring}

In the WAPPS integrity monitoring, an ideal zero mean Gaussian distribution is used to bound the residuals' non-zero mean Gaussian distribution. It ensures that the miss alert probability is less than the integrity risk (Wang and $\mathrm{Li}$ 2013). The algorithm uses the user differential range error of pseudo range (UDRE_PR) and differential range error of carrier phase (UDRE_PH) as system integrity monitoring parameters, which represent the differential errors of the pseudo range and carrier phase, respectively.

Limited by the WAPPS design, the correction covariance cannot be obtained so that it is impossible to calculate the UDREs by amplifying the variances directly. In this algorithm, the real-time residual statistics is measured for the UDREs computation, by using data from the monitoring stations. In addition, the alert judgment is carried out by the threshold comparison and Chi-square test. The process flow is shown in Fig. 5 and some key points are described below.
For each satellite, the residuals of all visible stations are used for real-time statistics so that UDRE_PR and UDRE_PH can be calculated by one-dimensional distribution parameters (Wang et al. 2015) according to Eq. (2), where $\mu$ and $\sigma$ are the mean and standard deviation and $\mathrm{K}$ is the Gaussian quantile corresponding to the miss alert rate.

$$
\begin{aligned}
& U D R E \_P R=\left(\left|\mu_{P R}\right|+K_{P R} \cdot \sigma_{P R}\right) / K_{P R} \\
& U D R E \_P H=\left(\left|\mu_{P H}\right|+K_{P H} \cdot \sigma_{P H}\right) / K_{P H}
\end{aligned}
$$

- UDRE_PR and UDRE_PH of each satellite calculated in the precedent epoch are used as thresholds in the current epoch. An alert flag will be set on the satellite whose residual exceeds the threshold. Then, all flags are used to vote and determine the correction's integrity status, including "Alert," "No Alert," and "Not Monitor."

- The influence of ambiguity could be eliminated in the calculation of the carrier phase residual. The precise coordinate of stations and continuous observation are used to achieve time smoothing, which can reduce the influence of ambiguity and station clock error. When the residuals error is less than a certain threshold, the ambiguity is con- 


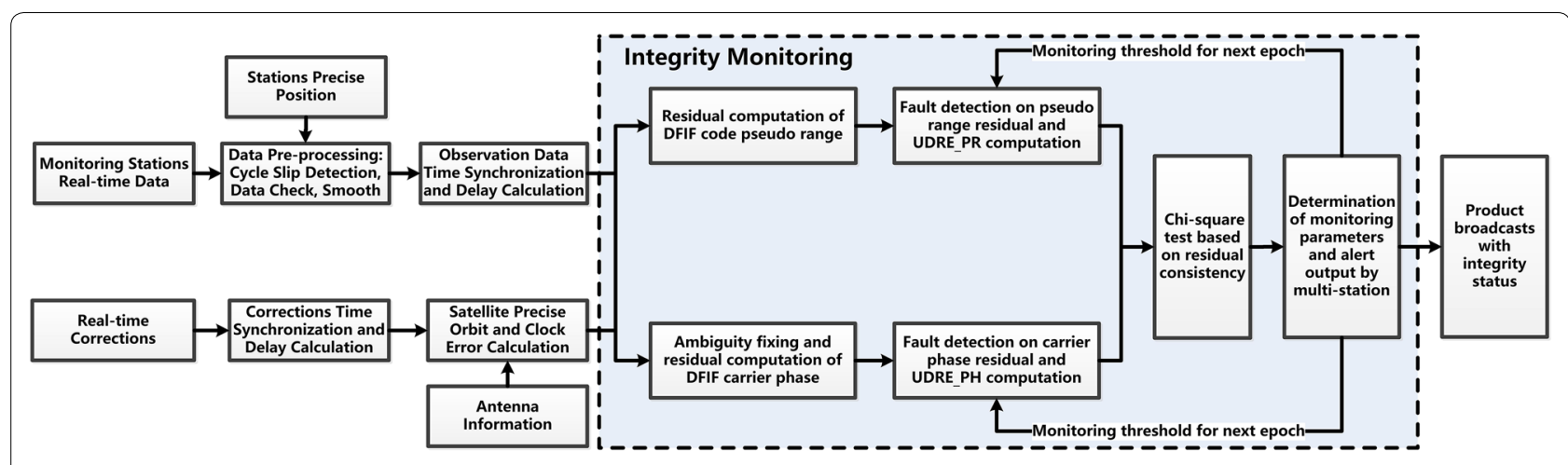

Fig. 5 Data flow of integrity monitoring for WAPPS

sidered to be fixed. As shown in Eq. (3), ph_resi is the carrier phase residual, $a m b$ is ambiguity, and $T$ is smooth length. This method can fix the ambiguity of all visible satellites individually, but the precision is limited by the influences of the measurement noise, the station clock error, and the DFIF's wavelength, which may affect the minimal detectable bias (MDB). Furthermore, AMB_Thread is set as a prior fixed value and the miss and false alert rate are not taken into consideration, which will be improved in the future.

$$
\begin{aligned}
& a m b(t)=p h \_r e s i(t) / T+a m b(t-1) \cdot(T-1) / T \\
& a m b(t)-a m b(t-1)<A M B \_T h r e a d
\end{aligned}
$$

- Using an independent and identical distribution of the satellites' residuals of each station, the algorithm uses a Chi-square test referring to the advanced receiver autonomous integrity monitoring (ARAIM) (EU-U.S. Cooperation on Satellite Navigation Working Group C, ARAIM Technical Subgroup 2012). As shown in Eq. (4), $y$ is the residual vector, $W$ is the weighting matrix, $G$ is the observation matrix, $N_{s a t}$ is the number of visible satellites, $N_{s y s}$ is the GNSS being used, $P_{F A}$ is the false alert rate, $T H$ is the threshold, and $\chi_{N}^{2}(\cdot)^{-1}$ is the inverse function of the Chi-square distribution function with $N$ degrees of freedom. $W$ relates to the correction's accuracy and measurement noise, which can be obtained by a regular evaluation. Note that $y$ includes both the pseudo range and carrier phase residuals computed by a known station position and clock error, instead of the carrier phase smoothed pseudo range residuals, which is computed in positioning in ARAIM, and the freedom degree of Chi Square is set as $N_{\text {sat }}$.

$$
\begin{aligned}
& \chi^{2}=y^{T}\left(W-W G\left(G^{T} W G\right)^{-1} G^{T} W\right) y \\
& T H=\chi_{N_{s a t}}^{2}\left(1-P_{F A}\right)^{-1}
\end{aligned}
$$

\section{Realization of the integrity monitoring}

As shown in Fig. 6, an in-house network with 20 stations in China (shown as red triangles) are used to implement integrity monitoring, while another 3 evaluation stations (shown as green triangles) are used to verify the performance.

Under the normal system status, the integrity monitoring performance is verified by comparing the evaluation the stations' pseudo range and carrier phase residuals with the integrity threshold. Generally, UDRE_PR and UDRE_PH should bound the residuals with a certain probability and ensure that the probability of the absolute value of the residual exceeding the threshold is less than the required miss alert rate.

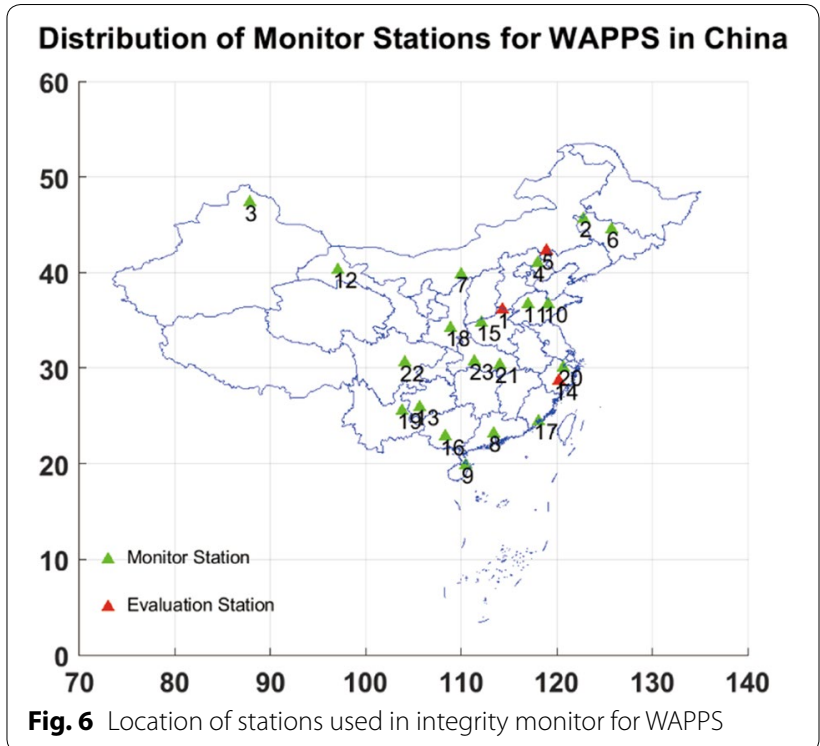


Figures 7 and 8 show the pseudo range and carrier phase monitoring results in the WAPPS. In both figures, the left subgraph shows the GPS corrections and the right one shows the BDS corrections, respectively. A blue point represents that no alarm epoch and a red point is an alert epoch. There are residuals of all visible satellites from three evaluation stations in one day. The data sampling is $1 \mathrm{~s}$, and the monitoring miss alert rate is $10^{-3}$.
Under the normal status, the integrity monitoring can ensure that the thresholds bound the corresponding residuals and the miss alert rate meets the requirement. At the same time, there is a false alert rate in $10^{-4}$ magnitude in carrier phase monitoring, which may affect the continuity of service and should be mitigated in the future.
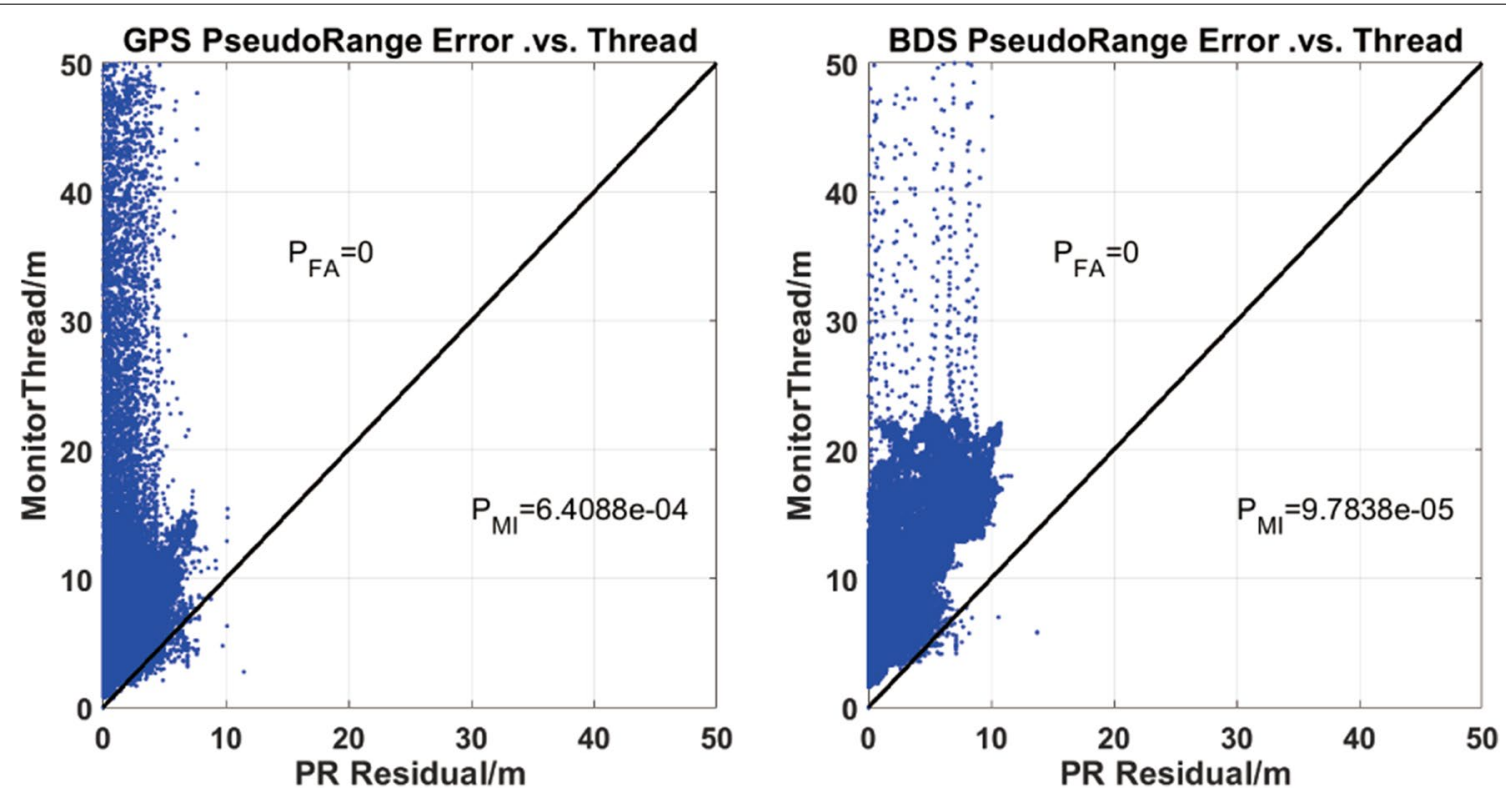

Fig. 7 WAPPS integrity monitor by pseudo range residual under normal status
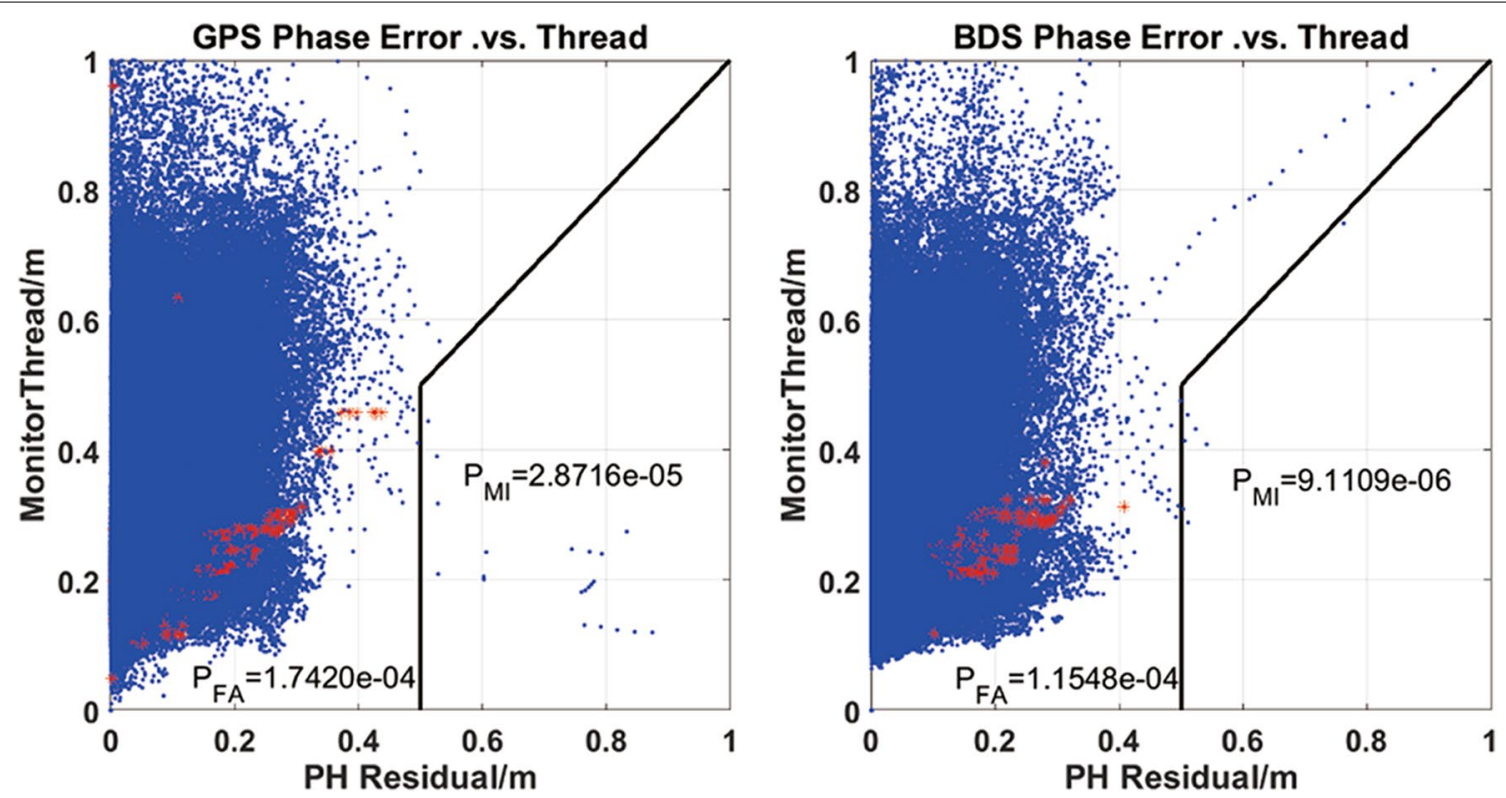

Fig. 8 WAPPS integrity monitor by carrier phase residual under normal status 
- Pseudo range monitoring is affected by the measurement noises with higher miss alert rate. In addition, because all monitoring stations are located in China, the elevation change when the satellite enters and exits China affects the threshold, evidently for the pseudo range threshold of the GPS.

- The carrier phase monitoring is almost unaffected by the entry and exit of satellites because of the process of ambiguity fixing, although the monitoring duration is reduced. Considering the correction accuracy, the absolute value of the phase residual is used, which is set to normal when it is less than $0.5 \mathrm{~m}$. As the result, the miss alert area in the carrier-monitoring diagram is different from that in the pseudo range one. There is also a miss and false alert epoch, mainly in the initial and final stages of ambiguity fixing, resulting from the decline of measurement quality.

\section{Fault simulation and verification Step fault monitoring}

The step fault is simulated by adding a step error on the corrections at certain epoch. To simplify the simulation, faults are added only on clock corrections. A single-fault model is used, while a step fault occurs only on one satellite in both GPS and BDS at the same time. The performance is verified by changing the fault value, such as 0.1 ,
$0.3,0.5,1$, and $5 \mathrm{~m}$, and the miss alert rate is set to be $10^{-3}$.

All the testing results are shown in Fig. 9. The top and bottom figures display the pseudo range and carrier phase monitoring, respectively, and only the results $30 \mathrm{~s}$ before and after the fault are shown. The red line is the epoch at the time when the fault happens, and the other lines are monitoring results. The positive value is UDREs and the negative value is alert. To verify the performance on the different orbit types of the satellites, MEO (GPS PRN01) and GEO (BDS PRN01) are selected as examples. The following conclusions are drawn.

- In pseudo range monitoring, the MDB is approximately $5 \mathrm{~m}$. The performance of carrier phase monitoring is better, the MDB of the MEO corrections is $0.5 \mathrm{~m}$ and that of the GEO corrections is $0.3 \mathrm{~m}$.

- The algorithm can ensure the alert being issued immediately when step faults occur, while the timeto-alert could be less than $3 \mathrm{~s}$, which can meet the need of a kinematic user.

\section{Slow drift fault monitoring}

Because the monitoring threshold is based on UDREs of the previous epoch, which increase with the slow drift of fault and cannot detect the anomaly effectively. Using a

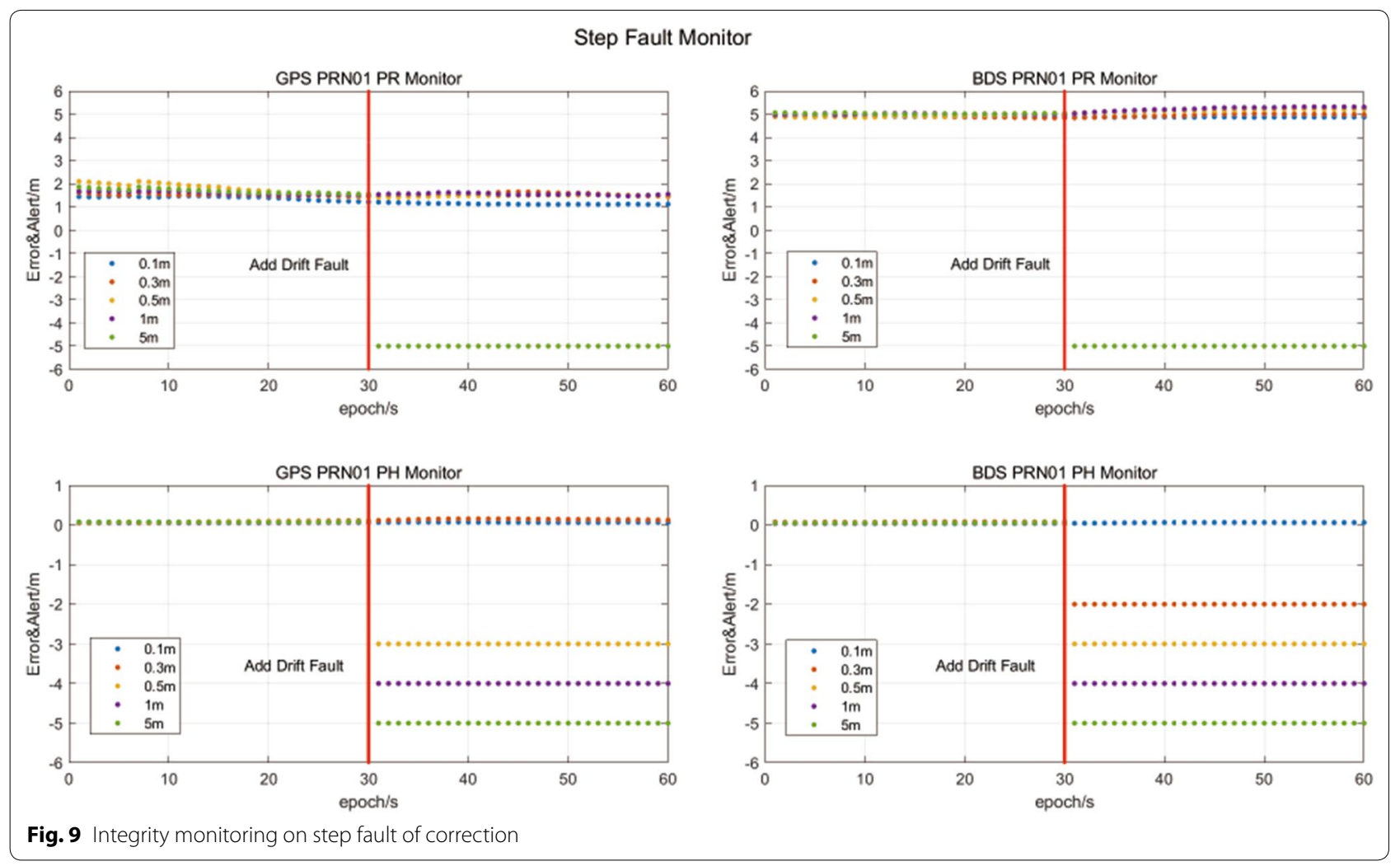


Chi-square test, the slow drift fault can be monitored by comparing the sum of squares of residuals with the Chisquare threshold.

The slow drift fault is added from certain epoch with various drift rates. Due to the correction accuracy, there is jitter with a rate of approximately $0.002 \mathrm{~m} / \mathrm{s}$ so that the false alert occurs easily if the simulation drift rate is set too low. Therefore, the rates are set as 0.005, 0.01, 0.05, 0.1 , and $0.5 \mathrm{~m} / \mathrm{s}$, and the false alert rate is set as $10^{-5}$.

The results of the slow drift fault tests are shown in Fig. 10. Results of $30 \mathrm{~s}$ before and after the fault are displayed. The red line is the epoch at the time when the fault happens and the others represent the monitoring results of different drifts. The positive value is UDREs while the negative values present alerts. Through the test results, the following conclusions are drawn.

- The pseudo range monitoring is insensitive to drift faults. When the drift rate is $0.5 \mathrm{~m} / \mathrm{s}$, an alert can be given in $9 \mathrm{~s}$ after a fault occurs. At this time, the offset is approximately $5 \mathrm{~m}$, which is equivalent to the step fault that it can detect.

- The carrier phase monitoring can detect a drift fault above $0.005 \mathrm{~m} / \mathrm{s}$ effectively. The time-to-alert also shortens with the increased drift rate. It can send an alert in $5 \mathrm{~s}$ with drift rate of $0.1 \mathrm{~m} / \mathrm{s}$ to ensure that the offset is less than $0.5 \mathrm{~m}$.
- Although a Chi-square test can monitor the drift fault, the parameters used must be set in accordance with the correction accuracy. If not, a false or miss alert might happen and affect the integrity and continuity of service. Thus, it is necessary to evaluate the corrections regularly.

\section{Conclusions}

In this study, the WAPPS integrity monitoring is presented. Real-time GPS and BDS corrections of an inhouse system and the station data are used to establish error models and to achieve the monitoring. Tests are conducted under both normal status and fault simulation conditions.

In normal status, the miss alert rate is below $10^{-3}$ and the false alert rate is approximately $10^{-4}$. In fault simulation, the monitoring can send a timely alert when a step or drift fault occurs, and the time-to-alert is less than $10 \mathrm{~s}$. The MDB of the pseudo range and carrier phase monitoring is of 5 and $0.5 \mathrm{~m}$, respectively. Moreover, a drift rate of $0.1 \mathrm{~m} / \mathrm{s}$ can be detected in $5 \mathrm{~s}$ by carrier phase monitoring.

The following work on the WAPPS integrity monitoring will focus on reducing the miss alert and false alert rates, as well as on shortening the time-to-alert. For improving the monitoring efficiency and effectiveness, risk models

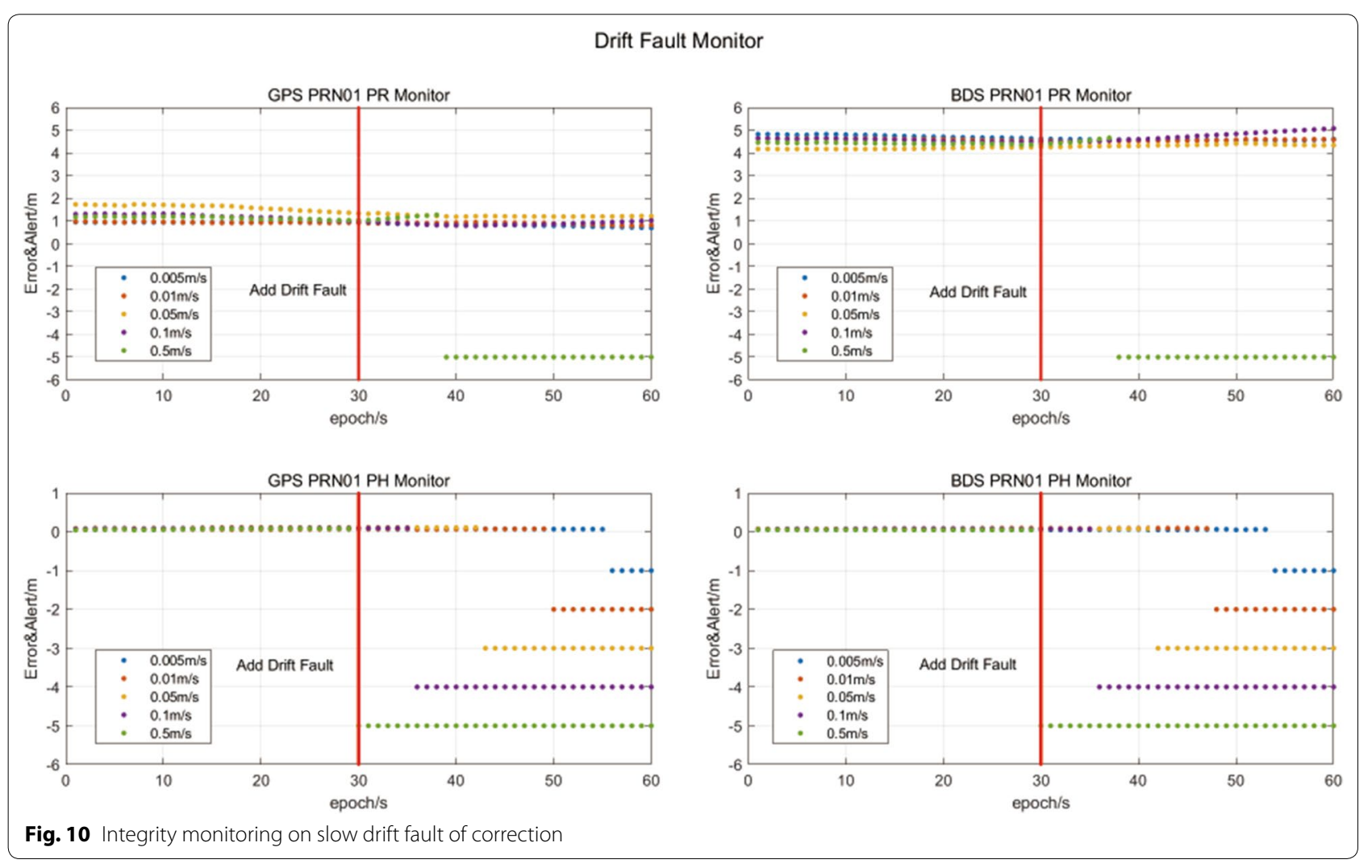


corresponding to various fault modes should be studied more deeply. Furthermore, the analysis and decomposition of the integrity requirement will be researched with extensive range of data.

\begin{abstract}
Abbreviations
WAPPS: Wide area precise positioning system; GNSS: Global navigation satellite system; PPP: Precise point positioning; UAV: Unmanned aerial vehicle; GFZ: Deutsches GeoForschungsZentrum; SISE: Signal-in-space error; DFIF: Dualfrequency ionosphere-free; UDRE PR: User differential range error of pseudo range; UDRE_PH: Differential range error of carrier phase; MDB: Minimal detectable bias; ARAIM: Advanced receiver autonomous integrity monitoring.
\end{abstract}

\section{Acknowledgements}

Not applicable.

\section{Authors' contribution}

YCW completed the architecture and algorithm design, system achievement, and evaluation and was a major contributor in writing the manuscript. JS provided data sources, definitions of monitoring, and architecture designs. All authors read and approved the final manuscript.

\section{Funding}

Not applicable.

\section{Availability of data and materials}

The data of WAPPS products and monitoring stations that support the findings of this study are available from Beijing UniStrong Science and Technology Corporation Limited. However, restrictions apply to the availability of these data, which were used under license for the current study, and thus, are not publicly available. Data are however available from the authors upon reasonable request and with permission of Beijing UniStrong Science and Technology Corporation Limited. The data of GFZ's products used to evaluate WAPPS performance during the current study are available in the Deutsches GeoForschungsZentrum repository, ftp://gfz-potsdam.de.

\section{Competing interests}

The authors declare that they have no competing interests.

Received: 10 August 2019 Accepted: 6 May 2020

Published online: 12 October 2020

\section{References}

Blanch, J. (2003). Using kriging to bound satellite ranging errors due to the ionosphere. Dissertation, Leland Stanford Junior University, America.

Blanch, J., Choi, M., \& Walter, T. (2010). Prototyping advanced RAIM for vertical guidance. In Proceedings of the 23rd international technical meeting of the satellite division of the institute of navigation (pp. 285-291).

Braff, R. (1997). Description of the FAA's Local Area Augmentation System (LAAS). Journal of the Institute of Navigation, 44(4), 411-423.

Brown, R. (1992). A baseline RAIM scheme and a note on the equivalence of three RAIM methods. In Proceedings of the 1992 national technical meeting of the Institute of Navigation (pp. 127-137).

Chen, G. C., Hu, Z. G., Wang, G. X., Chen, G., Liu, Z. Y., Zhao, Q. L. (2015) Assessment of BDS signal-in-space accuracy and standard positioning performance during 2013 and 2014. In China satellite navigation conference (CSNC) 2015 Proceedings (Vol. I, pp. 437-453).

Collins, P. (2008). Isolating and estimating undifferenced GPS integer ambiguities. In Proceedings of the 2008 National Technical Meeting of the Institute of Navigation (pp. 720-732).

Cui, H. Z., Tang, G. S., Song, B. Y., Liu, H. C., \& Ge, M. R. (2015). BDS satellite real time orbit and clock determination and initial results analysis. Chinese Space Science and Technology, 35(5), 1-7.

EU-U.S. Cooperation on Satellite Navigation Working Group C, ARAIM Technical Subgroup. (2013). Interim report, issue 1.0. https://www.gps.gov/polic y/cooperation/europe/2013/working-group-c/.

Ge, M., Gendt, G., Rothacher, M., Shi, C., \& Liu, J. (2008). Resolution of GPS carrier-phase ambiguities in Precise Point Positioning (PPP) with daily observations. Journal of Geodesy, 82(7), 389-399.

Kouba, J., \& Hérous, P. (2001). Precise point positioning using IGS orbit and clock products. GPS Solutions, 5(2), 12-28.

Laurichesse, D., Mercier, F., Berthias, J., Bijac, J. (2008). Real time zero difference ambiguities blocking and absolute RTK. In Proceedings of the 2008 National Technical Meeting of the Institute of Navigation (pp. 747-755).

Luo, M., Pullen, S., Dennis, J., Konno, H., et al. (2003). LAAS ionosphere spatial gradient threat model and impact of LGF and airborne monitoring. In Proceedings of the 16th international technical meeting of the satellite division of the institute of navigation (pp. 2255-2274).

Niu, F., Cai, Z. W., \& Feng, X. (2007). Discussion on project of decimeter-level wide area precise point positioning. Journal of Geomatics Science and Technology, 24(6), 439-442.

Parkinson, B., \& Axelrad, P. (1988). Autonomous GPS integrity monitoring using the pseudorange residual. ION, 35(2), 255-274.

Qu, L. Z., Zhao, Q. L., Li, M., \& Guo, J. (2013). Precise point positioning using combined BeiDou and GPS observations. In China satellite navigation conference (CSNC) 2013 proceedings (pp. 241-252).

Sayim, I. (2003). Ranging error overbounds for navigation integrity of local area augmented GPS. Dissertation, Illinois Institute of Technology, America.

Shi, C., Lou, Y. D., Song, W. W., \& Cai, H. (2009). A wide area real-time differential GPS prototype system and the initial results. Geomatics and Information Science of Wuhan University, 34(11), 1271-1274.

Tsai, Y.-J. (1999). Wide area differential operation of the global positioning system: Ephemeris and clock algorithms. Dissertation, Leland Stanford Junior University, America.

US Department of Defense. (2008, September). Global positioning system standard positioning service performance standard (4th edition).

Walter, T., Blanch, J., Phelts, R. E., \& Enge, P. (2012). Evolving WAAS to serve L1/L5 users. Journal of the Institute of Navigation, 59(4), 317-327.

Wang, Y. C., \& Li, R. (2013). The analysis of character of user range accuracy. In China satellite navigation conference (CSNC) 2013 proceedings (pp. 267-277).

Wang, Y. C., Li, R., \& Zhao, R. B. (2015). Research of signal-in-space integrity monitoring based on inter-satellite links. Chinese Journal of Electronics, 24(2), 439-444.

Zumberge, J. F., Heflin, M. B., Jefferson, D. C., Watkins, M. M., \& Webb, F. H. (1997). Precise point positioning for the efficient and robust analysis of GPS data from large networks. Journal of Geophysical Research, 102(B3), 5005-5017.

\section{Publisher's Note}

Springer Nature remains neutral with regard to jurisdictional claims in published maps and institutional affiliations. 